\title{
Rapid diagnostic tests to improve treatment of malaria and other febrile illnesses: patient randomised effectiveness trial in primary care clinics in Afghanistan
}

\begin{abstract}
Objective To assess the impact of rapid diagnostic tests on the diagnostic accuracy and treatment of malaria and non-severe fever in an Asian setting.

Design Patient randomised trial in primary level clinics.

Setting Two areas of Afghanistan where Plasmodium vivax and Plasmodium falciparum are endemic; one area with moderate transmission (eastern region) and one with low transmission (northern region).

Participants 5794 patients of all ages with suspected malaria enrolled by 80 clinicians in 22 clinics.

Interventions Malaria rapid diagnostic tests were compared with clinical diagnosis where no parasite diagnostic test was available, longer established field microscopy, and recently introduced microscopy.

Main outcome measures Proportion of patients appropriately treated with an antimalarial, defined as patients with $P$ vivax who received chloroquine, patients with $P$ falciparum who received artemisinin based combination therapy, and patients with no malaria parasites who did not receive an antimalarial. Secondary outcomes included diagnostic test accuracy and the proportion of patients negative for malaria who received antibiotics and antimalarials.

Results In the low transmission area, comparing rapid diagnostic tests with clinical diagnosis, $65 \%(212 / 325)$ versus $12 \%$ (40/321) of febrile patients were appropriately treated for malaria (adjusted odds ratio 92.7, $95 \%$ confidence interval 12.4 to $694.1, \mathrm{P}<0.001$ ). The proportion of patients who were negative for malaria and received an antibiotic was $57 \%(185 / 325)$ in the rapid diagnostic test arm compared with $14 \%$
\end{abstract}

(46/321) in the clinical diagnosis arm $(16.9,3.8$ to $75.4, \mathrm{P}<0.001)$. In the comparison of rapid diagnostic test with microscopy in the moderate transmission area, 83.6\% (1696/2028) versus 76.3\% (1512/1983) of patients were appropriately treated for malaria $(1.70,1.30$ to 2.23 , $\mathrm{P}<0.001)$. A higher proportion of $P$ falciparum cases received appropriate treatment with artemisinin based combination therapy when malaria was diagnosed by rapid diagnostic test $(82 \%, 58 / 71 v 32 \%, 24 / 76 ; 9.2,3.88$ to $21.66, \mathrm{P}<0.001)$.

Conclusions In South and central Asian regions of low to moderate malaria transmission where clinics lack capacity for diagnosis with rapid diagnostic tests or microscopy, the introduction of the tests should be considered to improve clinical care, reduce the overuse of antimalarials, and improve disease surveillance.

\section{Introduction}

Malaria is a major public health problem throughout South and central Asia, and approximately 2 billion people are at risk in areas with endemic disease. ${ }^{1-3}$ Despite the population at risk, compared with most of sub-Saharan Africa malaria in Asia is a less common cause of acute illness than are viral or bacterial infections, and most cases of malaria are due to Plasmodium vivax, which is rarely fatal. Falciparum malaria is comparatively rare in many areas, and thus fewer deaths from malaria occur among febrile patients in Asia than among those in Africa. Within the public health system, studies in India ${ }^{4}$ and Afghanistan ${ }^{5}$ show a widespread perception that non-specific febrile illness is largely due to malaria. As a result, substantial overdiagnosis of malaria and consequent mistreatment of 
non-malarial causes of fever with antimalarials is common. In a recent observational study in Afghanistan, 99\% of febrile patients who received a clinical diagnosis of malaria but were later shown not to have malaria parasites were given an antimalarial, and in other studies in India and Afghanistan where microscopy was used, $20 \%$ to $39 \%$ of those who tested negative for malaria were given antimalarials. ${ }^{45}$ Therefore the scope for improving the provision of appropriate treatment is considerable. The effect of rapid diagnostic tests for malaria on treatment of acute fevers in Africa has been extensively evaluated, but few investigations have been carried out in South and central Asia where the major proportion of malaria is due to $P$ vivax.

Rapid diagnostic tests for malaria could potentially be used in Asia to improve the targeting of antimalarials to true cases of malaria and to distinguish cases of falciparum malaria from cases of vivax malaria for artemisinin based combination therapy. ${ }^{6}$ Rapid diagnostic tests are increasingly used where parasitological diagnosis by microscopy is absent or of poor quality. In trials in Africa, the tests have shown clear advantages over diagnosis based on clinical signs and symptoms but have less often shown clear advantages over microscopy. ${ }^{7}$ Several trials have identified a problem of over-treatment with antimalarial drugs regardless of whether rapid diagnostic tests or microscopy are used. Unless rapid diagnostic tests are introduced with appropriate guidance and training, attempts to change clinicians' prescribing practices have proved slow at best. ${ }^{8-15}$ For patients who do not have malaria, the failure to consider other potential causes of fever can have serious consequences if left untreated. Artemisinin based combination therapy is expensive to source and if such treatment is wasted on patients who do not need it, the cost effectiveness of diagnosis and treatment is reduced. ${ }^{16} 17$

The epidemiology, transmission, and age of presentation of malaria is so different in Asia compared with Africa that clear evidence for the utility and impact of rapid diagnostic tests is needed before they are routinely deployed in the public sector. Basing the decision on the results of operational trials in Africa would be unwise and potentially unsafe for justifying such a major policy change. In most of Africa, rapid diagnostic tests only have to detect falciparum malaria. In Asia they need to distinguish vivax malaria from falciparum malaria and these tests are harder to use and have been less widely assessed. The treatments for $P$ vivax and $P$ falciparum require different drugs, so distinguishing between the species is essential for the provision of accurate treatment. The adoption of rapid diagnostic tests in Afghanistan and other South Asian countries is a major undertaking currently being considered without strong local evidence for effectiveness. Throughout the World Health Organization eastern Mediterranean region, policy makers are focusing on the expansion of malaria diagnosis using rapid diagnostic tests but lack trial evidence to make informed decisions.

In an earlier study in the same clinics as the current trial, ${ }^{5}$ routine microscopy failed to detect the few $P$ falciparum cases occurring among 2400 febrile patients, and only one of the six cases received the standard artemisinin based combination therapy (sulfadoxine-pyrimethamine with artesunate). Detecting falciparum malaria among other causes of fever, and targeting treatment accurately, is therefore a major challenge that acquires additional importance in countries on the northern margins of transmission that are hoping to achieve malaria elimination because success depends on capacity for accurate detection and reporting of cases.

Broadly two strategies exist: introducing rapid diagnostic tests for clinicians to guide their use of antimalarials where no diagnostic tests currently exist, and replacing or complementing microscopy with rapid diagnostic tests where microscopy diagnosis is currently used. We undertook a randomised trial to examine the effect of the introduction of malaria rapid diagnostic tests capable of detecting both vivax and falciparum malaria on prescribing behaviour in three settings that were representative of current practice: an area of low transmission, where clinicians based their diagnosis solely on clinical signs and symptoms; an area of low transmission, where microscopy had been introduced recently; and an area of moderate transmission, where routine microscopy was longer established.

\section{Methods}

\section{Study area and sites}

The study was conducted in Afghanistan in an area of moderate malaria transmission (in the eastern region adjacent to Pakistan) and an area of low malaria transmission (in the northern region adjacent to Tajikistan). Twenty two clinics were purposively selected as study sites in the two regions. In the low transmission area, where fewer than 1 in 100 malaria slides are positive, there were five clinics with no microscopy based diagnosis where clinicians used only clinical signs and symptoms, and five clinics with recently established microscopy. In the moderate transmission area, where about $20 \%$ to $30 \%$ of slides are positive (of which $90-95 \%$ show $P$ vivax and the remainder $P$ falciparum), there were 12 clinics where microscopy had been established for at least 10 years. Further details of the study areas have been reported previously. ${ }^{5}$

Clinicians from the selected clinics gave informed consent to participate in the study. A clinician was defined as health staff who consulted with patients and prescribed treatment; these included doctors, nurses, midwives, and community health supervisors.

\section{Patient enrolment and randomisation}

The clinician screened patients presenting at the clinics and enrolled those who gave informed consent and matched the inclusion criteria of current fever or self reported history of fever in the past 48 hours where the clinician suspected malaria and would normally request a diagnosis or would treat with a malaria drug. We excluded patients if they had a diagnostic result from another health facility; if the clinician provided treatment without testing (in facilities with testing available); if, for clinical reasons, the clinician specifically requested a blood slide before randomisation (for example, if parasite counts were requested). Written informed consent was taken by the clinician before randomisation.

Patients were randomised 1:1 to either the control or intervention arm. One of the investigators (AM) generated the randomisation sequences on 15 September 2009 using block sizes of 8 to 12 for each individual clinic and the open source statistics package, $\mathrm{R}$ (www.r-project.org). The allocation was printed on individual cards showing the patient number and clinic. These were stored at each clinic in individual opaque envelopes and opened by the clinician after informed consent was given. Once the envelope was opened, the patient was deemed to have entered the study. Assessors of trial laboratory outcomes (reference microscopists and laboratory technologists) were blind to the allocation of the patient.

\section{Control and intervention description}

The control group in each clinic represented the currently available diagnostic method for malaria, which was clinical 
diagnosis in five of the clinics in the low transmission area, recently introduced microscopy in the five remaining clinics in that area, and microscopy in all 12 clinics in the moderate transmission area. Trained technicians carried out microscopy in the clinics. No refresher training was done before the study. Typically, the microscopists examined thick and thin blood smears after staining slides with 3\% Giemsa stain, and examined 100 fields on the thick film before declaring a slide as negative.

The intervention was the use of a malaria rapid diagnostic test for diagnosis. Initially, CareStart Pf (HRPII)/Pv (pLDH) (AccessBio, NJ) were used, but these were replaced with CareStart Pf (HRPII)/Pan (pLDH) (AccessBio, NJ) following the adoption of the latter as the nationally recommended test midway through the trial. ${ }^{18}$ Training on the use and interpretation of the tests followed the national training package and was given to study clinicians and nursing staff in each clinic together with a bench aid. Rapid diagnostic tests were provided to each clinic at the start of the trial and replenished when required.

\section{Data and specimen collection}

Data were collected on individually labelled case record forms. Clinicians noted the patients' symptoms, their diagnosis, and any treatment given. Before treatment, the study registrar took a reference blood slide from the patients. Whatman 3MM chromatography paper (Whatman, $\mathrm{NJ}$ ) was used to collect blood spots from each patient and stored with desiccant for later processing by polymerase chain reaction.

\section{Laboratory analysis}

Study registrars prepared the reference blood slides in the clinics. Thick and thin smears were prepared according to standard operating procedures. Thin smears were fixed with methanol and the slide was allowed to air dry before being stored in a slide box. Within one week, two trained reference microscopists who were blinded to each other's results and the allocation of the patient double read the slides using light microscopy. The reference microscopists used standard solutions of 3\% Giemsa to stain the slides. They defined a slide as negative if no parasites were seen after examining 100 fields. If the slide showed a positive result, the microscopists counted and recorded the asexual parasite counts against 200 white cells. A third microscopist who was blinded to the original diagnoses read the slides that were discordant for the presence or absence of parasites or for Plasmodium species, and a best of three rule applied.

The final reference diagnosis for malaria was blood slides with concordant results between the two microscopists, and if the slide results differed, the diagnosis was based on polymerase chain reaction of filter paper blood samples. ${ }^{19}{ }^{20}$ Polymerase chain reaction was used to confirm all microscopy blood films with a positive result and a random $10 \%$ of blood films with a negative result.

\section{Trial outcomes}

The primary outcome was the proportion of patients appropriately treated for suspected malaria measured against the reference diagnosis. Appropriate treatment is a composite outcome based on local treatment guidelines and defined as: patients with $P$ vivax receiving chloroquine, patients with $P$ falciparum or mixed infections receiving artemisinin based combination therapy (sulfadoxine/pyrimethamine-artesunate), and patients who are negative for malaria and not given an antimalarial. To make it clearer for clinicians we changed the way the primary outcome was presented in this paper from the original trial protocol, which was the proportion of patients inappropriately treated. This is equivalent to 1 minus the proportion appropriately treated, as presented here.

Secondary outcomes were analysis of treatment accuracy disaggregated by infection type (the proportions of patients with $P$ vivax receiving chloroquine, patients with $P$ falciparum receiving artemisinin based combination therapy, and patients with no parasites receiving no antimalarial drugs), analysis of the accuracy of treatment against the clinic based test (that is, appropriate prescribing based on the clinic's laboratory diagnosis by rapid diagnostic test or microscopy), and the proportion of patients receiving antibiotics. The accuracy of microscopy and the rapid diagnostic tests was also compared with the reference diagnosis.

\section{Sample size and data analysis}

Sample sizes were based on showing superiority for the primary outcome between rapid diagnostic tests and the existing diagnostic methods in each setting — that is, in the moderate transmission region (microscopy versus rapid diagnostic test); in the low transmission region (microscopy versus rapid diagnostic test and clinical diagnosis versus rapid diagnostic test). The sample size calculations based on previous data assumed the proportion of patients appropriately treated were $10 \%$ when based on clinical diagnosis and $60 \%$ when based on microscopy, one clinician per clinic, and variability between clinics of half of the mean effect of the diagnostic test on the outcome. At least 710 patients in clinics in the moderate transmission area and 312 in the low transmission area using microscopy were required to detect, with $80 \%$ power, a $20 \%$ increase of appropriate treatment using rapid diagnostic tests compared with microscopy. Fifty patients in the low transmission clinical diagnosis setting were required to detect, with $80 \%$ power, a $70 \%$ increase of appropriate treatment using rapid diagnostic tests. ${ }^{21}$ All sample size calculations assumed a significance level of $95 \%(\alpha=0.05)$. To account for seasonality in malaria and the possibility that the use and perception of rapid diagnostic tests may change over time, recruitment continued for a full malaria season and beyond the minimum sample size.

Unadjusted estimates of the primary outcome were provided for each study setting (clinical diagnosis versus rapid diagnostic test or microscopy versus rapid diagnostic test) and region (moderate or low transmission). We estimated the crude effect of the diagnostic method separately: in the low transmission setting, clinical diagnosis versus rapid diagnostic test and microscopy versus rapid diagnostic test; and in the moderate transmission area, microscopy versus rapid diagnostic test.

We used a three level mixed effect model identically applied to each region/setting to adjust for clustering by clinician and clinics. To allow the intervention effect to vary across clinicians, we fitted clinician and clinic as random effects and study arm as a random slope. We fitted a priori covariates (age and sex of patient, positivity of clinic slide, clinician's qualification, and type of clinic) as fixed effects in the models. The variation between clinics and clinicians was assessed through the likelihood ratio test. Secondary outcomes were analysed using the methods previously described.

Within each control-intervention group we used $\chi^{2}$ tests to compare the accuracy of microscopy and rapid diagnostic tests to the ideal reference diagnosis, but we did not adjust for clustering. Statistical analysis was conducted using STATA version 13.0 and the analysis plan was approved by the data and safety monitoring board before analysis. 


\section{Results}

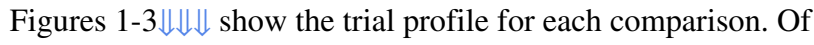
62005 patients seen in the outpatient departments of the clinics, 5749 were eligible and enrolled in the trial between 23 September 2009 and 22 September 2010.

Outcomes were available and analysed for a total of 5695 patients (99\%). Data from 54 patients were not included in the analysis owing to undefined reference results (unusable slides or filter papers). Those excluded were equally distributed among the clinics and between the study arms. Table $1 \Downarrow$ shows enrolment characteristics at the patient and clinic level for each arm and area. Within each region, patients in the two arms were similar but in the low transmission setting the age of those randomised to rapid diagnostic test was lower than those randomised to the clinical diagnosis arm.

Table $2 \Downarrow$ shows the results of the reference malaria diagnosis. The proportion of patients infected did not differ between arms in any area, but, as expected, fewer cases occurred in the low transmission area than in the moderate transmission area. Cases of falciparum malaria were only seen in the moderate transmission area.

\section{Rapid diagnostic tests compared with clinical diagnosis}

In the low transmission area, the proportion of patients receiving appropriate malaria treatment was higher in the rapid diagnostic test arm $(212 / 325,62 \%)$ than in those with a diagnosis based on signs and symptoms alone $(40 / 321,12 \%$; odds ratio 13.2 , $95 \%$ confidence interval 8.8 to $19.8, \mathrm{P}<0.001)$. The effect strengthened after adjusting for clustering using the multilevel model (adjusted odds ratio 92.7, 95\% confidence interval 12.4 to 684.1, $\mathrm{P}<0.001)$. The effect of rapid diagnostic tests on treatment varied highly between clinics $\left(\chi^{2}=\mathrm{P}<0.001\right.$, figs $4-6 \Downarrow \Downarrow \Downarrow)$. Because no malaria cases were reported from these five clinics (table 2) all of the antimalarial drugs prescribed were to patients who were negative for malaria. A higher proportion of patients with a negative rapid diagnostic test result received an antibiotic compared with patients in the clinical diagnosis arm $(185 / 325,57 \%$ v 46/321, 14\%; 16.9, 3.8 to 75.4 , $\mathrm{P}<0.001)$

\section{Rapid diagnostic tests compared with microscopy}

In the moderate transmission area, the proportion of patients receiving appropriate malaria treatment in the rapid diagnostic test arm increased marginally compared with the microscopy $\operatorname{arm}(1696 / 2028,83.6 \% v 1512 / 1983,76.3 \% ; 1.70,1.30$ to 2.23 , $\mathrm{P}<0.001)$.

Among the patients with a reference diagnosis of $P$ falciparum, those with a diagnosis by rapid diagnostic test were more likely to be treated with an artemisinin based combination therapy than those with a diagnosis by microscopy $(58 / 71,82 \% v 24 / 76$, $32 \%$; $9.2,3.88$ to $21.66, \mathrm{P}<0.001$, table $3 \Downarrow)$. Patients with a reference diagnosis of $P$ vivax were as likely to be treated with chloroquine (the recommended treatment) regardless of whether the diagnosis was by rapid diagnostic test or by microscopy ( $>90 \%$ in the moderate transmission area).

Half of all antimalarials prescribed in the moderate transmission area were given to patients who were negative for malaria, including 41 (33\%) of the 123 doses of artemisinin based combination therapy used during the study. Overall, this misprescription did not differ between arms, but more treatments with artemisinin based combination therapy were prescribed to patients in the rapid diagnostic test arm than those in the microscopy arm (86 doses $v 37$ doses) because more cases of falciparum malaria were detected by rapid diagnostic tests than by microscopy (table $4 \Downarrow$ ). The chance of patients with a negative malaria result by rapid diagnostic test or microscopy being treated with an antibiotic was similar (796/1561, 51.0\% v $709 / 1473,48.2 \% ; 1.11,0.88$ to $1.41, \mathrm{P}=0.34)$.

Patients with a negative reference diagnosis were less likely to receive an antimalarial in the rapid diagnostic test arm $(292 / 1561,18.7 \%)$ than in the microscopy arm $(378 / 1473$, $25.7 \% ; 1.68,1.26$ to $2.24, \mathrm{P}<0.001$, table 3 ).

In the low transmission area, a modest but significant difference was seen in treatment accuracy when rapid diagnostic tests were compared with microscopy $(418 / 521,80.2 \%$ v 389/510, 76.3\%; $1.73,1.08$ to $2.78, \mathrm{P}=0.022$, table 3 ), and the proportion of patients negative for malaria who received an antibiotic was similar between the rapid diagnostic test and microscopy arms (411/521, 78.8\% v 387/510, 74.1\%; $1.33,0.95$ to $1.85, \mathrm{P}=0.1)$. Whether rapid diagnostic tests or microscopy was used, $99.8 \%$ of malaria treatments were given to those who did not have malaria.

\section{Prescriber response to available test results}

In the moderate transmission area, use of rapid diagnostic tests did not reduce over-treatment with antimalarials compared with microscopy when prescribers were faced with a negative test result $(187 / 1478,12.7 \% \vee 197 / 1345,14.7 \% ; 0.78,0.53$ to 1.17 , $\mathrm{P}=0.23$ ), and a similar result was observed in the low transmission area $(86 / 448,19.2 \% v 99 / 517,19.2 \%, 0.94,0.53$ to $1.67, \mathrm{P}=0.84)$. In northern clinics with no microscopy, negative rapid diagnostic test results resulted in 35\% (112/324) of patients receiving an antimalarial.

\section{Diagnostic accuracy of microscopy and rapid diagnostic tests}

Microscopy had a lower operational sensitivity for detection of $P$ falciparum than either of the rapid diagnostic test types used in the study (table 4$)$. Of 43 patients with a false negative diagnosis of $P$ falciparum by microscopy, 21 (49\%) were misdiagnosed as having $P$ vivax, all of whom received chloroquine treatment, and $22(51 \%)$ were misdiagnosed as being negative for malaria, 21 of whom received no antimalarial. Of the 21 untreated $P$ falciparum cases, 12 (55\%) received an antibiotic, some of which may have antimalarial properties. ${ }^{22}$ Also, among the $P$ falciparum cases there was no evidence of a difference in parasite density between those who were true positives and those who were false negatives: median parasite density 130 (interquartile range 63.5-752) p/uL v 204 (45-724) $\mathrm{p} / \mathrm{uL}$, Wilcoxon rank sum test $\mathrm{P}=0.8$.

\section{Discussion}

Overdiagnosis and over-treatment of malaria is a major problem in South and central Asia where malaria is a minority cause of febrile illness, and primary health centres often rely on clinical symptoms for a diagnosis of malaria. Introducing rapid diagnostic tests for malaria with a realistic, scalable training package showed clear advantages over clinical diagnosis for reducing overdiagnosis and improving the targeting of antimalarials. Clinical diagnosis of malaria is unreliable and clinicians are unable to distinguish malaria from other causes of fever. ${ }^{23}$ Blanket treatment for suspected malaria with no parasite confirmation is no longer supportable, and expansion of effective diagnosis is needed. 
When compared with routine microscopy, rapid diagnostic tests were more consistently accurate and led to higher detection rates of falciparum malaria resulting in improved treatment with artemisinin based combination therapy. Rapid diagnostic tests did not reduce the overuse of antimalarials in patients with a negative test result compared with microscopy. Clinicians may see a positive blood film as confirmation of their clinical judgment, whereas a negative diagnosis is still considered to be suspected malaria. ${ }^{24}$ This may, in part, stem from a lack of faith in negative rapid diagnostic test or microscopy results, a product of long held practices and beliefs that "most dangerous fever is malaria," and the fear of missing a case of malaria. ${ }^{15}$ In moderate transmission settings effective diagnosis distinguishes the potentially fatal falciparum malaria from the more common vivax malaria. In both settings effective diagnosis provides reliable surveillance data on local transmission trends and gives clinicians a more realistic perspective on the local importance of malaria.

\section{Implications for policy and practice}

One disadvantage of rapid diagnostic tests is that they do not provide a quantitative measure of parasite density. In some settings in Africa where low parasite levels may not be clinically relevant, the lack of a parasite count may lead to a diagnosis of malaria but also to other causes of fever being missed. This is not likely to be true in most of Asia, including Afghanistan where there is little appreciable immunity to clinical malaria.

A full economic analysis taking into account local transmission settings will be needed for local policy makers - there may be transmission settings below which rapid diagnostic tests are not $\operatorname{cost}_{\text {effective }}{ }^{16}$ - but the clinical case for rapid diagnostic tests where there is no microscopy is clear. Improving microscopy is an alternative to using rapid diagnostic tests but is difficult to maintain. In general, microscopists who are poorly trained or poorly motivated will return results of low sensitivity and specificity (not just low sensitivity). Improving field microscopy and maintaining accuracy requires regular monitoring of clinics and quality control by supervisory microscopists. ${ }^{25}$ Sustained inputs can lead to improvements in diagnosis and surveillance, but the maintenance of quality control can easily be affected by shortfalls in resources..$^{25}$

Tackling the diagnosis and treatment of malaria can be seen as essential components of the larger problem of improving the treatment of febrile illness. Most of the patients in our study did not have malaria, and many alternative causes of febrile disease occur in Afghanistan, only some of which require antimicrobials. ${ }^{26}$ Patients who are negative for malaria are often treated with antibiotics as an alternative to antimalarials, but identifying which of these patients really need this treatment is essential to help reduce antibiotic resistance pressure and wastage. This study suggests that there is a risk of substituting the over-prescription of antimalarials with over-prescription of antibiotics. Although some of the patients who get antibiotics will benefit from them, the global pressure of antimicrobial resistance means that blind antibiotic treatment of all febrile illness should be discouraged.

\section{Limitations of this study}

A potential limitation of any trial of clinician behaviour is the Hawthorne effect, where awareness of being observed may change behaviour or practice. Individually randomised trials (as opposed to a cluster randomised design) may also underestimate effects where clinicians' influence one another. The major strengthening of the adjusted odds ratio compared with the unadjusted odds ratio in the comparison of clinical diagnosis with rapid diagnostic test was not driven by patient level confounding factors such as age but occurred in the multilevel model. This suggests a strong influence of clinicians on each other's practice within clinics, which is known to be a major driver of prescriber behaviour. ${ }^{15}$

Overall, a lower proportion of consultations in the moderate transmission area than in the low transmission area resulted in enrolment into the trial (figs 1-3). This reflects several contextual factors: differing uptake and use of diagnostic services between settings, with services being relatively new in the low transmission setting, and differing healthcare usage among the population. Clinicians were given the sole decision to enrol patients, and assessing whether patients were appropriately identified for malaria diagnosis was not an objective. Although not measured directly, clinicians' perception of local transmission and the introduction of diagnostics seem to influence the decision to test for malaria. In the low transmission area, the relatively recent introduction of diagnosis and the perception that malaria is a common cause of fever is reflected in the higher proportion of patients enrolled and receiving a diagnostic test. It is only when microscopy is well established as in the moderate transmission area that clinicians see a low proportion of positive cases and consequently this reduces the proportion of patients who are referred for diagnosis.

The study setting can be generalised to areas of South and central Asia, where vivax and falciparum malaria coexist, diagnostic services are weak or absent, and the population remains at risk of malaria. The only other study that tested for malaria found substantial overdiagnosis and over-treatment with antimalarials in India. ${ }^{4}$

\section{Conclusions}

With simple training, the introduction of rapid diagnostic tests for malaria for use in patients with fever showed clear advantages over clinical diagnosis and some advantages over microscopy in primary level clinics in two epidemiological settings, improving the accuracy of treatment of vivax and falciparum malaria. However, the adoption of rapid diagnostic tests also increased antibiotic treatment for non-malarial febrile illness compared with microscopy or clinical diagnosis and did not reduce over-prescription of antimalarials among patients with a negative test result.

As part of the expansion of diagnostic services in low resource South Asian settings the inclusion of rapid diagnostic tests for malaria should be considered, especially where clinical diagnosis is widespread. Because non-malarial causes of febrile illness will be identified, implementation should also include training on rational prescription of both antimalarials and antibiotics. The adoption of rapid diagnostic tests in areas where endemic disease is low is as important as their adoption in areas of substantial transmission.

We thank Baptiste Leurent (ACT Consortium, London School of Hygiene and Tropical Medicine) for advice on the analysis, Asif Alokozai (Health Protection and Research Organisation) for assistance with data processing, and Cyril Buhler (Health Protection and Research Organisation) for advice on interpretation of the polymerase chain reaction; the data safety monitoring board and the trial steering committee in Afghanistan, including the national malaria and leishmaniasis control programme and World Health Organization, Roll Back Malaria focal point; the ACT Consortium Secretariat at London School of Hygiene and Tropical Medicine; and the input of all involved 


\section{What is already known on this topic}

Both Plasmodium vivax and Plasmodium falciparum malaria exist in South and west Asia, with vivax the major species Over-treatment of vivax malaria is common and $P$ falciparum malaria often goes undetected

Trials of rapid diagnostic tests for malaria in Africa, where falciparum malaria predominates, were only partially effective at reducing overtreatment of malaria

\section{What this study adds}

In Afghanistan rapid diagnostic tests for malaria reduced inappropriate antimalarial use compared with clinical diagnosis The tests also improved the detection and treatment of rare cases of $P$ falciparum malaria compared with microscopy $20-30 \%$ of patients are treated for malaria despite having a negative test result

clinic staff, trial field staff, laboratory staff, and managers of HealthNet TPO, Merlin, and the Health Protection and Research Organisation.

The trial was prospectively registered at ClinicalTrials.gov

(NCT00935688) on 8 July 2009. An amendment to the registration was made on 13 August 2009 to include full details of the protocol.

Contributors: TL designed the study, directed the project in the field, conducted the analysis, and wrote the paper. AM codesigned the study, managed the project, conducted the laboratory analysis, and contributed to the paper. IM assisted in the study design, managed and supervised the field work and data, and contributed to the paper. BC codesigned and conducted the statistical analysis. MA, SHB, and NM assisted in the study design, acted as regional managers, assisted with analysis, and contributed to the paper. RZ and HuR conducted the laboratory testing, interpreted the results, and contributed to the paper. CJMW codesigned the study, analysed data, and revised the paper. MR conceived of and codesigned the study, was project leader, and contributed to the data analysis, writing of the paper and revisions. TL is the guarantor.

Funding Source: The study was funded by the Bill and Melinda Gates Foundation though a grant to the ACT Consortium at the London School of Hygiene \& Tropical Medicine. The funding source played no role in the design, conduct, analysis or interpretation of the results.

Competing interests: All authors have completed the ICMJE uniform disclosure form at www.icmje.org/coi_disclosure.pdf and declare: the research was funded by the Bill and Melinda Gates Foundation and Sponsored by the London School of Hygiene and Tropical Medicine; no financial relationships with any organisations that might have an interest in the submitted work in the previous three years; no other relationships or activities that could appear to have influenced the submitted work.

Ethical approval: This trial was approved by the institutional review board, Ministry of Public Health, Afghanistan and the ethics committee of the London School of Hygiene and Tropical Medicine.

Data sharing: The full dataset is available from the corresponding author at toby.leslie@lshtm.ac.uk.

Transparency: The lead author (TL) affirms that the manuscript is an honest, accurate, and transparent account of the study being reported; that no important aspects of the study have been omitted; and that any discrepancies from the study as planned (and, if relevant, registered) have been explained.

World Health Organization. World malaria report. WHO, 2012

2 Guerra CA, Howes RE, Patil AP, Gething PW, Van Boeckel TP, Temperley WH, et al. The international limits and population at risk of Plasmodium vivax transmission in 2009. PLoS Negl Trop Dis 2010;4:e774.

3 Hay SI, Okiro EA, Gething PW, Patil AP, Tatem AJ, Guerra CA, et al. Estimating the global clinical burden of Plasmodium falciparum malaria in 2007. PLoS Med 2010;7:e1000290.

4 Joshi R, Colford JM Jr, Reingold AL, Kalantri S. Nonmalarial acute undifferentiated fever in a rural hospital in central India: diagnostic uncertainty and over-treatment with antimalarial agents. Am J Trop Med Hyg 2008;78:393-9.
5 Leslie T, Mikhail A, Mayan I, Anwar M, Bakhtash S, Nader M, et al. Overdiagnosis and mistreatment of malaria among febrile patients at primary healthcare level in Afghanistan: observational study. BMJ 2012;345:e4389.

6 World Health Organization. Universal access to malaria diagnostic testing: an operational manual. WHO, 2011.

7 Bastiaens GJH, Bousema T, Leslie T. Scale-up of malaria rapid diagnostic tests and artemisinin-based combination therapy: challenges and perspectives in Sub-Saharan Africa. PLOS Med 2014;11:e1001590.

8 Ansah EK, Narh-Bana S, Epokor M, Akanpigbiam S, Quartey AA, Gyapong J, et al. Rapid testing for malaria in settings where microscopy is available and peripheral clinics where only presumptive treatment is available: a randomised controlled trial in Ghana. BMJ 2010;340:c930.

9 Nankabirwa J, Zurovac D, Njogu JN, Rwakimari JB, Counihan H, Snow RW, et al. Malaria misdiagnosis in Uganda—implications for policy change. Malar J 2009;8:66.

10 Ngasala B, Mubi M, Warsame M, Petzold MG, Massele AY, Gustafsson LL, et al. Impact of training in clinical and microscopy diagnosis of childhood malaria on antimalarial drug prescription and health outcome at primary health care level in Tanzania: a randomized controlled trial. Malar J 2008;7:199.

11 Reyburn H, Ruanda J, Mwerinde O, Drakeley C. The contribution of microscopy to targeting antimalarial treatment in a low transmission area of Tanzania. Malar J 2006;5:4.

12 McMorrow ML, Masanja MI, Abdulla SM, Kahigwa E, Kachur SP. Challenges in routine implementation and quality control of rapid diagnostic tests for malaria-Rufiji District, Tanzania. Am J Trop Med Hyg 2008;79:385-90.

13 Moonasar D, Goga AE, Frean J, Kruger P, Chandramohan D. An exploratory study of factors that affect the performance and usage of rapid diagnostic tests for malaria in the Limpopo Province, South Africa. Malar J 2007;6:74.

14 Reyburn H, Mbakilwa H, Mwangi R, Mwerinde O, Olomi R, Drakeley C, et al. Rapid diagnostic tests compared with malaria microscopy for guiding outpatient treatment of febrile illness in Tanzania: randomised trial. BMJ 2007;334:403.

15 Chandler Cl, Jones C, Boniface G, Juma K, Reyburn H, Whitty CJM. Guidelines and mindlines: why do clinical staff over-diagnose malaria in Tanzania? A qualitative study. Malar J 2008;7:53.

16 Lubell $\mathrm{Y}$, Reyburn H, Mbakilwa H, Mwangi R, Chonya K, Whitty CJM, et al. The cost-effectiveness of parasitologic diagnosis for malaria-suspected patients in an era of combination therapy. Am J Trop Med Hyg 2007;77:128-32.

17 Lubell Y, Reyburn H, Mbakilwa H, Mwangi R, Chonya S, Whitty CJM, et al. The impact of response to the results of diagnostic tests for malaria: cost-benefit analysis. BMJ 2008;336:202-5.

18 Mikhail AF, Leslie TJ, Mayan MI, Zekria R, Mohammad N, Hasanzai MA, et al. Field trial of three different Plasmodium vivax-detecting rapid diagnostic tests with and without evaporative cool box storage in Afghanistan. Malar J 2011:10:169.

19 Shokoples SE, Ndao M, Kowalewska-Grochowska K, Yanow SK. Multiplexed real-time PCR assay for discrimination of Plasmodium species with improved sensitivity for mixed infections. J Clin Microbiol 2009;47:975-80.

20 Mangold KA, Manson RU, Koay ES, Stephens L, Regner M, Thomson RB Jr, et al. Real-time PCR for detection and identification of Plasmodium spp. J Clin Microbiol 2005:43:2435-40

21 Brown H, Prescott R. Applied mixed models in medicine. Wiley, 2004

22 Dahl EL, Rosenthal PJ. Multiple antibiotics exert delayed effects against the Plasmodium falciparum apicoplast. Antimicrob Agents Chemother 2007;51:3485-90.

23 Chandramohan D, Carneiro I, Kavishwar A, Brugha R, Desai V, Greenwood B. A clinical algorithm for the diagnosis of malaria: results of an evaluation in an area of low endemicity. Trop Med Int Health 2001:6:505-10.

24 Reynolds J, Wood M, Mikhail A, Ahmad T, Karimullah K, Motahed M, et al. Malaria "diagnosis" and diagnostics in Afghanistan. Qual Health Res 2013;23:579-91.

25 Rowland M, Rab MA, Freeman T, Durrani N, Rehman N. Afghan refugees and the temporal and spatial distribution of malaria in Pakistan. Soc Sci Med 2002;55:2061-72.

26 Wallace MR, Hale BR, Utz GC, Olson PE, Earhart KC, Thornton SA, et al. Endemic infectious diseases of Afghanistan. Clin Infect Dis 2002;34:S171-207.

Accepted: 13 May 2014

\section{Cite this as: BMJ 2014;348:g3730}

This is an Open Access article distributed in accordance with the Creative Commons Attribution Non Commercial (CC BY-NC 3.0) license, which permits others to distribute, remix, adapt, build upon this work non-commercially, and license their derivative works on different terms, provided the original work is properly cited and the use is non-commercial. See: http://creativecommons.org/licenses/by-nc/3.0/. 


\section{Tables}

Table 1 | Characteristics of clinics and patients enrolled in the trial, by area and study arm. Values are number (percentages) unless stated otherwise

\begin{tabular}{|c|c|c|c|c|c|c|}
\hline \multirow[b]{2}{*}{ Characteristics } & \multicolumn{2}{|c|}{ Moderate transmission (east region) } & \multicolumn{4}{|c|}{ Low transmission (north region) } \\
\hline & Microscopy arm & RDT arm & Microscopy arm & RDT arm & Clinical arm & RDT arm \\
\hline No of patients excluded from analysis & 22 & 20 & 2 & 4 & 2 & 4 \\
\hline No of patients included in analysis & 1983 & 2027 & 515 & 523 & 321 & 325 \\
\hline Males & $1116(56.3)$ & $1073(52.9)$ & $274(53.2)$ & $281(53.7)$ & $187(58.3)$ & $187(57.5)$ \\
\hline Mean (SD) age (years) & $15.4(12.6)$ & $15.8(12.9)$ & $20.1(14.9)$ & $19.4(15.1)$ & $24.2(16.6)$ & $22.9(15.8)$ \\
\hline \multicolumn{7}{|l|}{ Age group: } \\
\hline $0-5$ & $368(18.6)$ & $349(17.2)$ & $101(19.6)$ & $102(19.5)$ & $24(7.5)$ & $35(10.8)$ \\
\hline $6-10$ & $598(30.2)$ & $601(29.6)$ & $57(11.1)$ & $74(14.2)$ & $37(11.5)$ & $32(9.9)$ \\
\hline $\begin{array}{lll}11-15 \\
\end{array}$ & $305(15.4)$ & $321(15.8)$ & $64(12.4)$ & $68(13.0)$ & $57(17.8)$ & $57(17.5)$ \\
\hline $16-20$ & $282(14.2)$ & $305(15.0)$ & $90(17.5)$ & $87(16.6)$ & $67(20.9)$ & $60(18.5)$ \\
\hline $21-40$ & $337(17.0)$ & $336(16.6)$ & $158(30.7)$ & $147(28.1)$ & $86(26.8)$ & $106(32.6)$ \\
\hline$>40$ & $93(4.7)$ & $116(5.7)$ & $45(8.7)$ & $45(8.6)$ & $50(15.6)$ & $35(10.8)$ \\
\hline \multicolumn{7}{|l|}{ Patients by season: } \\
\hline Summer & $524(26.4)$ & $545(26.9)$ & $172(33.4)$ & 169 (32.2) & $139(43.3)$ & $137(42.2)$ \\
\hline Autumn & $671(33.8)$ & $672(33.1)$ & $107(20.8)$ & $114(21.8)$ & $39(12.2)$ & $43(13.2)$ \\
\hline Winter & $323(16.3)$ & $334(16.5)$ & $76(14.8)$ & $80(15.3)$ & $40(12.5)$ & $38(11.7)$ \\
\hline Spring & $465(23.5)$ & $477(23.5)$ & $160(31.1)$ & $160(30.6)$ & $103(32.1)$ & $107(32.9)$ \\
\hline \multicolumn{7}{|l|}{ Diagnosed by RDT type: } \\
\hline $\mathrm{Pf} / \mathrm{Pv}+$ & - & $1691(83.5)$ & - & $376(72.3)$ & - & $204(62.8)$ \\
\hline Pf/Pant & - & $334(16.5)$ & - & $144(27.7)$ & - & $121(37.2)$ \\
\hline No of clinics & \multicolumn{2}{|c|}{12} & \multicolumn{2}{|c|}{5} & \multicolumn{2}{|c|}{5} \\
\hline \multicolumn{7}{|l|}{ Clinic type } \\
\hline Basic health centre & \multicolumn{2}{|c|}{5} & \multicolumn{2}{|c|}{5} & \multicolumn{2}{|c|}{5} \\
\hline Comprehensive health centre & \multicolumn{2}{|c|}{7} & \multicolumn{2}{|c|}{0} & \multicolumn{2}{|c|}{0} \\
\hline
\end{tabular}

No of clinics in each transmission level

(defined by slide positivity, \%):

\begin{tabular}{|c|c|c|c|c|c|c|}
\hline $0 \%$ & \multicolumn{2}{|c|}{0} & \multicolumn{2}{|c|}{1} & \multicolumn{2}{|c|}{5} \\
\hline$>0-3 \%$ & \multicolumn{2}{|c|}{0} & \multicolumn{2}{|c|}{4} & \multicolumn{2}{|c|}{0} \\
\hline $3-20 \%$ & \multicolumn{2}{|c|}{4} & \multicolumn{2}{|c|}{0} & \multicolumn{2}{|c|}{0} \\
\hline $21-25 \%$ & \multicolumn{2}{|c|}{4} & \multicolumn{2}{|c|}{0} & \multicolumn{2}{|c|}{0} \\
\hline$>25 \%$ & \multicolumn{2}{|c|}{4} & \multicolumn{2}{|c|}{0} & \multicolumn{2}{|c|}{0} \\
\hline Median (range) No of clinicians per clinic & $3(2-8)$ & $3(2-7)$ & $4(1-6)$ & $4(1-6)$ & $3(1-3)$ & $3(1-4)$ \\
\hline Median (range) No of patients per clinic & $165(120-229)$ & $169(127-231)$ & $103(77-136)$ & $105(76-134)$ & $64(32-116)$ & $65(28-122)$ \\
\hline $\begin{array}{l}\text { Median (range) No of patients per } \\
\text { clinician }\end{array}$ & $33(1-170)$ & $33(1-175)$ & $10(1-136)$ & $12(1-134)$ & $25(1-98)$ & $19(1-95)$ \\
\hline
\end{tabular}

RDT=rapid diagnostic test; Pf/Pv=CareStart Pf (HRPII)/Pv (pLDH) (AccessBio, NJ); Pf/Pan=CareStart Pf (HRPII)/Pan (pLDH) (AccessBio, NJ). 
Table 2| Number of patients with malaria diagnosis by double read reference blood smears and confirmed by polymerase chain reaction among sample, by location and study arm. Values are number of patients (\% of all cases) unless stated otherwise

\begin{tabular}{|c|c|c|c|c|c|c|}
\hline \multirow{2}{*}{$\begin{array}{l}\text { Malaria reference diagnosis } \\
\text { and species }\end{array}$} & \multicolumn{2}{|c|}{ Moderate transmission (east region) } & \multicolumn{4}{|c|}{ Low transmission north region } \\
\hline & $\begin{array}{l}\text { Microscopy arm } \\
(n=1983)\end{array}$ & RDT arm $(n=2028)$ & $\begin{array}{l}\text { Microscopy arm } \\
(n=515)\end{array}$ & RDT arm (n=523) & $\begin{array}{c}\text { Clinical arm } \\
(n=321)\end{array}$ & $\begin{array}{c}\text { RDT arm } \\
(n=325)\end{array}$ \\
\hline $\begin{array}{l}\text { No }(\%) \text { with reference slide } \\
\text { positive for malaria }\end{array}$ & $510(25.7)$ & $466(23.0)$ & $5(1.0)$ & $2(0.4)$ & 0 & 0 \\
\hline \multicolumn{7}{|l|}{ Composition of slide: } \\
\hline Vivax malaria & $434(85.1)$ & $395(84.8)$ & $5(100)$ & $2(100)$ & 0 & 0 \\
\hline Falciparum malaria & $75(14.7)$ & $69(14.8)$ & 0 & 0 & 0 & 0 \\
\hline Coinfection with both species & $1(0.2)$ & $2(0.4)$ & 0 & 0 & 0 & 0 \\
\hline
\end{tabular}


Table 3| Number, proportion, and relative odds of patients being appropriately treated after diagnosis of malaria in two transmission areas in east and north of Afghanistan

\begin{tabular}{|c|c|c|c|c|c|}
\hline Outcome measure by intervention arm & $\begin{array}{c}\text { No with outcome/No in } \\
\text { group (\%) }\end{array}$ & $\begin{array}{l}\text { Unadjusted odds ratio } \\
\qquad(95 \% \mathrm{Cl})\end{array}$ & $P$ value & $\begin{array}{l}\text { Adjusted odds ratio* } \\
\qquad(95 \% \mathrm{Cl})\end{array}$ & $P$ value \\
\hline \multicolumn{6}{|l|}{ Moderate transmission (east region) } \\
\hline \multicolumn{6}{|l|}{ Patients appropriately treated: } \\
\hline Microscopy & $1512 / 1983(76.3)$ & 1 & \multirow[t]{2}{*}{$<0.001$} & 1 & \multirow[t]{2}{*}{$<0.001$} \\
\hline RDT & 1696/2028 (83.6) & 1.59 (1.35 to 1.87$)$ & & 1.70 (1.30 to 2.23$)$ & \\
\hline \multicolumn{6}{|l|}{$\begin{array}{l}\text { Patients negative for malaria not given } \\
\text { antimalarial: }\end{array}$} \\
\hline Microscopy & $1095 / 1473(74.3)$ & 1 & & 1 & \\
\hline RDT & $1270 / 1562(81.3)$ & 1.50 (1.26 to 1.79$)$ & $<0.001$ & 1.68 (1.26 to 2.24$)$ & $<0.001$ \\
\hline \multicolumn{6}{|l|}{$\begin{array}{l}\text { Patients with Plasmodium vivax receiving } \\
\text { chloroquine: }\end{array}$} \\
\hline Microscopy & $393 / 434(90.6)$ & 1 & & 1 & \\
\hline RDT & 368/395 (93.2) & $1.42(0.86$ to 2.36$)$ & 0.17 & $1.52(0.90$ to 2.58$)$ & 0.12 \\
\hline \multicolumn{6}{|l|}{$\begin{array}{l}\text { Patients with Plasmodium falciparum } \\
\text { receiving artemisinin based combination } \\
\text { therapy: }\end{array}$} \\
\hline Microscopy & 24/76 (31.5) & 1 & & 1 & \\
\hline RDT & $58 / 71(81.7)$ & 7.6 (3.57 to 16.37$)$ & $<0.001$ & 9.2 (3.88 to 21.66$)$ & $<0.001$ \\
\hline \multicolumn{6}{|l|}{ Low transmission (north region) } \\
\hline \multicolumn{6}{|l|}{ Patients appropriately treated: } \\
\hline Microscopy & $393 / 515(76.3)$ & 1 & \multirow[t]{2}{*}{0.12} & 1 & \multirow[t]{2}{*}{0.022} \\
\hline RDT & $420 / 523(80.3)$ & $1.27(0.94$ to 1.70$)$ & & 1.73 (1.08 to 2.78$)$ & \\
\hline \multicolumn{6}{|l|}{$\begin{array}{l}\text { Patients negative for malaria not given } \\
\text { antimalarial: }\end{array}$} \\
\hline Microscopy & $389 / 510(76.3)$ & 1 & & & \\
\hline RDT & $418 / 521(80.2)$ & $1.26(0.94$ to 1.70$)$ & 0.12 & 1.66 (1.03 to 2.66$)$ & 0.036 \\
\hline \multicolumn{6}{|l|}{$\begin{array}{l}\text { Patients with } P \text { vivax receiving } \\
\text { chloroquine: }\end{array}$} \\
\hline Microscopy & $4 / 5(80.0)$ & - & - & 一 & 一 \\
\hline RDT & $2 / 2(100.0)$ & - & - & - & - \\
\hline \multicolumn{6}{|l|}{ Patients appropriately treated: } \\
\hline Clinical & $40 / 321(12.5)$ & 1 & \multirow[t]{2}{*}{$<0.001$} & 1 & \multirow[t]{2}{*}{$<0.001$} \\
\hline $\mathrm{RDT}$ & $212 / 325$ (65.2) & 13.2 (8.8 to 19.7$)$ & & 92.7 (12.4 to 694.1$)$ & \\
\hline
\end{tabular}

${ }^{*}$ Adjusted odds ratio using three level model, adjusted for fixed effect of patient's age and clinic type (in east region) and random effects of clinician (within clinics) and clinic (between clinics). Analysis for adjusted odds ratio is based on two level model of patients nested within clinicians and clinicians nested within clinics. 
Table 4| Accuracy of field microscopy and rapid diagnostic tests (RDT) used in trial for diagnosis of falciparum and vivax malaria against polymerase chain reaction confirmed reference blood slides

\begin{tabular}{|c|c|c|c|}
\hline \multirow[b]{2}{*}{ Performance measures by malaria type } & \multicolumn{3}{|c|}{ Diagnosis type } \\
\hline & Microscopy & Pf/Pv RDT & Pf/Pan RDT \\
\hline \multicolumn{4}{|l|}{ Diagnosis of falciparum malaria } \\
\hline Sensitivity \% (95\% Cl) & $43.4(41.2$ to 45.1$)$ & $93.8(92.6 \text { to } 94.9)^{*}$ & 85.7 (82.0 to 89.5$) \dagger$ \\
\hline No with diagnosis/No in group & $33 / 76$ & $60 / 64$ & $6 / 7$ \\
\hline Specificity \% (95\% Cl) & 99.0 (98.6 to 99.4$)$ & 98.1 (97.4 to 98.8$) \ddagger$ & 99.4 (98.6 to 100$)$ \\
\hline No with diagnosis/No in group & $1887 / 1906$ & $1595 / 1626$ & $325 / 327$ \\
\hline \multicolumn{4}{|l|}{ Diagnosis of vivax malaria } \\
\hline \multicolumn{4}{|l|}{ Moderate transmission area: } \\
\hline Sensitivity \% $(95 \% \mathrm{Cl})$ & 88.0 (86.6 to 89.5$)$ & $92.4(91.2$ to 93.7$) \S$ & $94.5,(92.1$ to 97.0$) \S$ \\
\hline No with diagnosis/No in group & $382 / 434$ & $281 / 304$ & $86 / 91$ \\
\hline Specificity \% $(95 \% \mathrm{Cl})$ & $86.9(85.4$ to 88.4$)$ & 94.6 (93.8 to 95.9$) \ddagger$ & 95.9 (93.8 to 98.0$) \ddagger$ \\
\hline No with diagnosis/No in group & $1345 / 1548$ & $1314 / 1386$ & $233 / 243$ \\
\hline \multicolumn{4}{|l|}{ Low transmission area: } \\
\hline Sensitivity \% $(95 \% \mathrm{Cl})$ & $80.0(76.4$ to 83.6$)$ & & \\
\hline No with diagnosis/No in group & $4 / 5$ & $0 / 2$ & 一 \\
\hline Specificity \% (95\% Cl) & 97.4 (95.9 to 98.8$)$ & $99.7(99.2 \text { to } 100)^{\star}$ & 97.9 (93.9 to 99.6$)$ \\
\hline No with diagnosis/No in group & $447 / 459$ & $373 / 374$ & $138 / 141$ \\
\hline \multicolumn{4}{|l|}{ Clinical diagnosis $\mathbb{Z}$} \\
\hline Specificity \% (95\% Cl): & 11.8 (8.5 to 15.9$)$ & 100 & $99.2(95.4 \text { to } 100)^{*}$ \\
\hline No with diagnosis/No in group & $38 / 321$ & $0 / 204$ & $119 / 120$ \\
\hline
\end{tabular}

Comparing microscopy with each type of RDT.

$\mathrm{Pf} / \mathrm{Pv}=$ CareStart Pf (HRPII)/Pv (pLDH) (AccessBio, NJ); Pf/Pan=CareStart Pf (HRPII)/Pan (pLDH) (AccessBio, NJ).

*Fisher's exact test, $P=0.01-<0.001$.

†Fisher's exact test, $\mathrm{P}=0.048$.

$\ddagger X^{2}=6.4, P=0.01-<0.001$ (2 degrees of freedom).

§Fisher's exact test, $\mathrm{P}=0.06-0.1$

ISensitivity not calculable as no reference slide positive cases were seen in clinical diagnosis setting. 


\section{Figures}

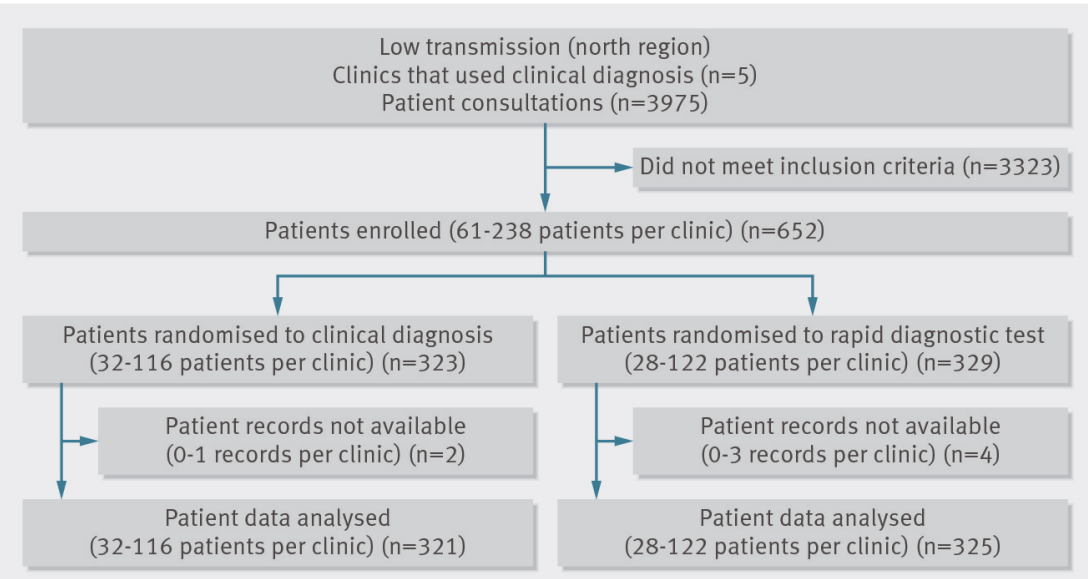

Fig 1 Patient flow through trial in low transmission area (north region) in five clinics that lacked microscopy and used clinical signs and symptoms to diagnose malaria

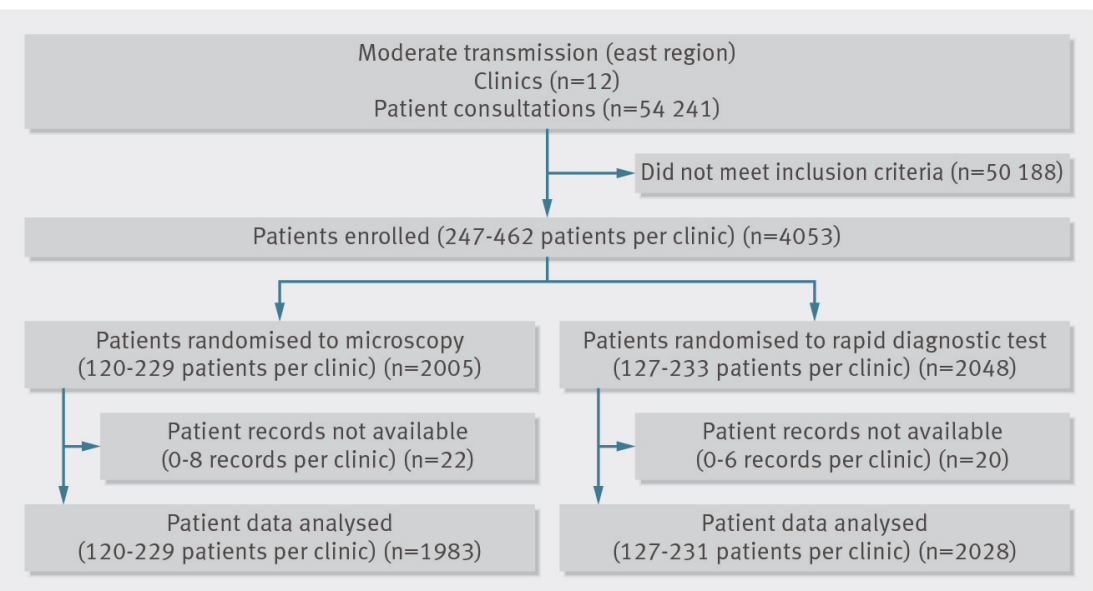

Fig 2 Patient flow through trial in moderate transmission area (east region) in 12 clinics that used microscopy to diagnose malaria

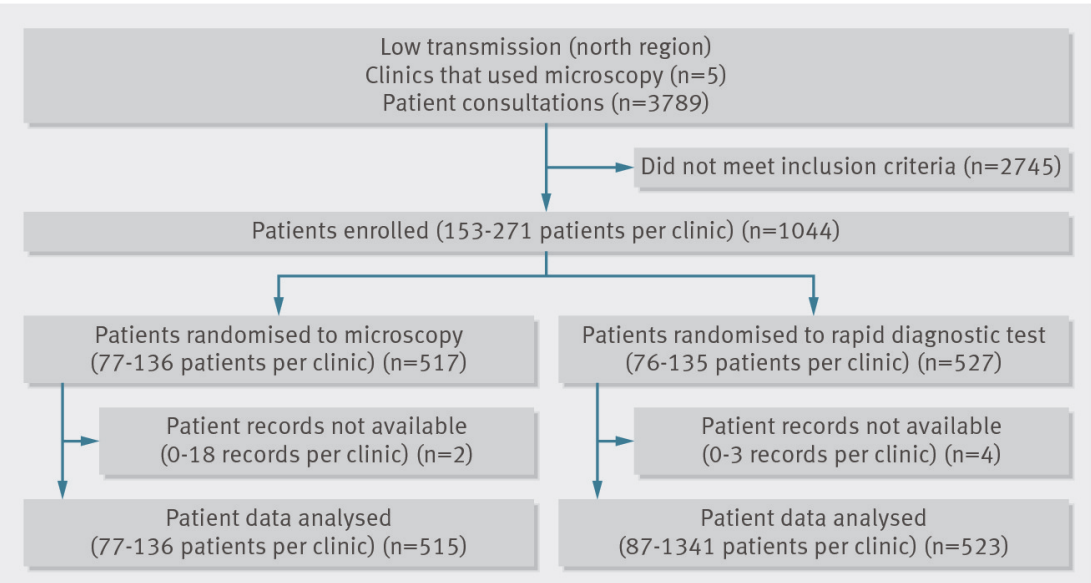

Fig 3 Patient flow through trial in low transmission area (north region) in five clinics that used microscopy to diagnosis malaria 


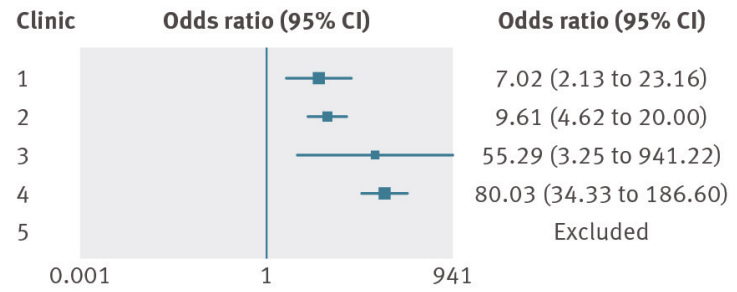

Fig 4 Forest plot showing relative odds for appropriate malaria treatment in clinics in low transmission area using clinical diagnosis versus rapid diagnostic test (RDT). Clinic 5 was excluded because of zero events (no patients appropriately treated in control arm)

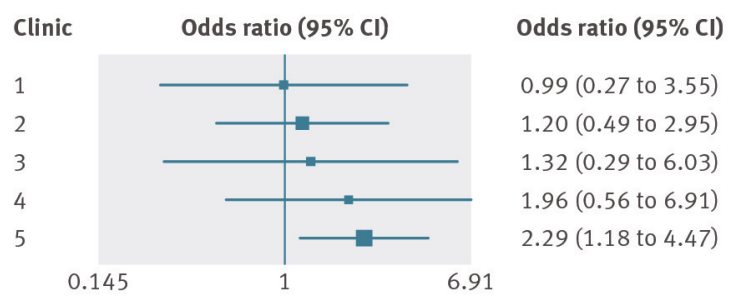

Fig 5 Forest plot showing relative odds for appropriate malaria treatment in clinics in low transmission area using microscopy versus rapid diagnostic test (RDT)

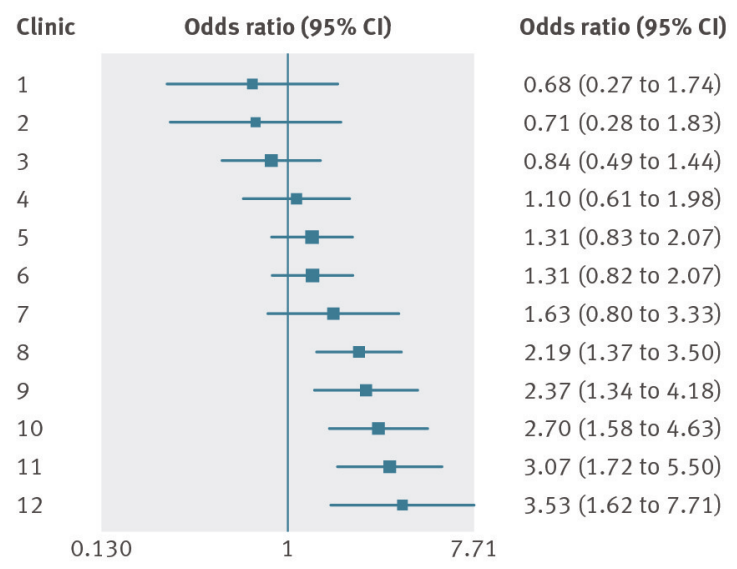

Fig 6 Forest plot showing relative odds for appropriate malaria treatment in clinics in moderate transmission area using microscopy versus rapid diagnostic test (RDT) 